\title{
Leveraging Health Information Exchange to Support Public Health Situational Awareness: The Indiana Experience
}

\author{
Shaun J. Grannis ${ }^{1,2}$, Kevin C. Stevens ${ }^{3}$, Ricardo Merriwether ${ }^{2}$ \\ ${ }^{1}$ The Regenstrief Institute \\ ${ }^{2}$ Indiana University School of Medicine \\ ${ }^{3}$ Marion County Health Department, Indiana
}

\section{Abstract}

Public health situational awareness is contingent upon timely, comprehensive and accurate information from clinical systems. Ad-hoc models for sending non-standard clinical information directly to public health are inefficient and increasingly unsustainable. Information sharing models that leverage Health Information Exchanges (HIEs) are emerging. HIEs standardize, aggregate and streamline information sharing among data partners, including public health stakeholders, and HIE has supported public health practice in Indiana for more than 10 years. To accelerate nationwide adoption of HIE-supported situational awareness processes, the CDC awarded three HIEs across the nation, including Indiana, New York and Washington/Idaho. The Indiana partners included Indiana University School of Medicine, Regenstrief Institute, Indiana Health Information Exchange, Indiana State Department of Health, Health \& Hospital Corporation of Marion County, and Children's Hospital Boston. Activities included augmenting biosurveillance processes, enabling bi-directional communication, enhancing automated detection of notifiable conditions, and demonstrating technological advances at national forums. HIE transactions destined for public health were enhanced with standardized clinical vocabulary and more complete physician contact information. During the $2009 \mathrm{H} 1 \mathrm{N1}$ flu outbreak, the HIE delivered targeted public health broadcast messages to providers in Marion County, Indiana. We will review the partnership characteristics, activities, accomplishments and future directions for our health information exchange.

Keywords: health information exchange, situational awareness, biosurveillance, syndromic surveillance, influenza.

\section{Introduction}

Under an initiative entitled "Accelerating Situational Awareness through Health Information Exchange" the Centers for Disease Control and Prevention (CDC) partnered with an Indiana coalition including the Indiana State Department of Health (ISDH), the Marion County Health Department (MCHD) in Indianapolis, the Regenstrief Institute (RI), Indiana University (IU), and Children's Hospital Boston. Coalitions from New York and Washington/Idaho also partnered with the $\mathrm{CDC}$, and the combined groups are collectively referred to as the "CDC $\mathrm{HIE}$ 
Collaborative." The CDC charged the collaborative with exploring methods for sharing information between public health and clinical entities to support public health situational awareness and case reporting in the context of the emerging Nationwide Health Information Network (NHIN). This paper summarizes the activities of the Indiana coalition.

\section{Partners}

The organizational characteristics of the contract participants are described below.

Regenstrief Institute, Inc. - In 1969 philanthropist Samuel N. Regenstrief established the Regenstrief Institute to conduct and facilitate medical research, medical education and clinical care. As a supporting organization of Indiana University, Regenstrief is an internationally recognized informatics and healthcare research organization dedicated to improving health through research that enhances the quality and cost-effectiveness of health care. The Institute employs approximately 150 full-time staff in addition to 35 investigators and affiliated researchers who are faculty members of Indiana University.

Indiana University School of Medicine - Indiana University's medical school was established in 1903 and is one of the nation's largest medical centers. The Indianapolis campus includes Indiana's only medical and dental schools and the nation's largest nursing school. IU Medical School supports three adult hospitals (University, Wishard, and Roudebush Veteran's), a pediatric hospital (James Whitcomb Riley Hospital for Children), a health center (Regenstrief Institute for Health Care) and a number of unique teaching and research facilities.

Indiana Health Information Exchange (IHIE) - In 2004 IHIE was incorporated in the state of Indiana as a non-profit company. IHIE is extending and scaling the principles and infrastructure devised, demonstrated, and built by the Regenstrief Institute. IHIE works hand-in-hand with Regenstrief to create sustainable business models and provide commercial support for the Institute's technologies in the marketplace. IHIE has grown more than 50 employees and continues to add new data sources and new customers each month. IHIE provides a clinical results delivery service called DOCS4DOCS $®$ that transmits more than 1.4 million electronic clinical results per month to over 19,000 physicians. Because clinical workflow and health information technologies are highly varied, the transmission of results is tailored to accommodate specific workflows by delivering results directly to EMR's, web portals, and other receipt mechanisms. Additional services that leverage the HIE infrastructure are being launched.

Indiana State Department of Health (ISDH) - The Indiana State Department of Health, founded as the state board of health in 1881, supports Indiana's economic prosperity and quality of life by promoting, protecting, and providing for the health of Hoosiers in their communities. In addition to providing epidemiological support to most of the local health departments in the state, ISDH offers a full complement of skills including epidemiology, information technology and program management. The Department's headquarters are located in downtown Indianapolis.

Health \& Hospital Corporation of Marion County (HHC) -- For over 50 years HHC of Marion County has served as the public health and hospital system for Marion County, Indiana. 
HHC operates the Marion County Health Department (MCHD) and Wishard Health Services (WHS). MCHD is Indiana's largest local health department and provides a variety of services to improve population and environmental health. WHS is the public hospital and healthcare system for uninsured and underinsured residents of Marion County. HHC is mandated by the Indiana General Assembly to provide medical services to all residents of Marion County, regardless of ability to pay. A seven-member board of trustees, appointed by the Mayor of Indianapolis, the City-County Council, and the County Commissioners, governs HHC. The director and most of the medical staff at the MCHD are faculty at the Indiana University School of Medicine.

Children's Hospital Boston (CHB) - Founded in 1869, CHB is home to the world's largest research enterprise based at a pediatric medical center. The Children's Hospital Informatics Program at the Harvard-MIT Division of Health Sciences and Technology (CHIP) is a core program of the Center for Biomedical Informatics at Harvard Medical School, and a core program of the NIH funded National Center for Biocomputing, a cornerstone of the NIH Roadmap Initiative. CHIP investigators lead several regional and national efforts in public health surveillance, data integration across multiple hospitals, and personally controlled health records.

\section{Activities Summary}

Activities supported by the CDC HIE Collaborative were grouped according to major tasks and included 1.) Biosurveillance, 2.) Technical demonstrations, 3.) Aggregate Summary Data Exchange, 4.) Delivering Public Health Alerts, 5.) Pre-populated Reporting Forms, 6.) Enhancing Automated Notifiable Condition Detection, and 7.) Improving Data Quality for Public Health. The following sections provide an overview of activities in each of these areas.

\section{Biosurveillance}

A number of successful activities helped to augment traditional public health surveillance.

Extracting public health concepts from free-text data: Many conditions of interest to public health are recorded in widely varying free-text formats. We developed natural language processing and other information extraction methods to identify concepts in non-standard freetext reports. Concepts that suggest the presence of a condition of interest to public health (e.g., the term "miliary" in a chest x-ray report suggests tuberculosis) can augment syndromic surveillance data streams.

Resource Utilization Monitoring: Hospital bed utilization data is often gathered by manual data entry, which further encumbers already overburdened personnel. We created a prototype process to evaluate the feasibility of characterizing hospital bed utilization using existing admission, discharge and transfer (ADT) messages received by the HIE. We confirmed the ability to gage bed utilization trends at participating hospitals in a predictable fashion using these transactions.

Assessing the Minimum Biosurveillance Data Set (MBDS): From 2006 to 2008 the American Health Information Community (AHIC), a national subject matter expert panel, established a Minimum Biosurveillance Data Set (MBDS) containing a representative collection of data elements to support public health surveillance processes. To assess the utility and feasibility of 
using this data set, we completed a technical review of the data elements by first mapping data fields from the existing HIE transactions to specific MBDS elements and second, we determined the percent completeness for each data element in the current operational data flow.

EMS and Inpatient Surveillance: While the HIE infrastructure continues to support the Indiana Public Health Emergency Surveillance System (PHESS) by delivering Minimum Biosurveillance Data Set (MBDS) elements from 83 Indiana hospitals, we also seek other useful surveillance sources. In addition to traditional surveillance we developed a strategy to exchange HIE data with Emergency Medical Services (EMS) mobile units, supporting surveillance in the prehospital phase. Developing additional EMS interfaces and incorporating additional data feeds from ambulatory care facilities are ongoing. To enhance Indiana's public health surveillance activities, in 2010 we began leveraging the HIE data network to collect inpatient chief complaint data and currently transmit this data to ISDH every 3-hours. Because we could leverage existing infrastructure, we were able to develop data feeds from more than 50 hospitals in less than 3 months.

Evaluating Open Source Syndromic Surveillance Tools: In partnership with Children's Hospital Boston, we locally deployed and evaluated an open source surveillance tool called Automated Epidemiologic Geotemporal Integrated Surveillance System (AEGIS). This work resulted in documents describing surveillance systems and a technical characterization of AEGIS.

\section{Technical Demonstrations}

In the face of newly created interoperability specifications there is a need to demonstrate the feasibility of deploying new technology. The CDC HIE Collaborative demonstrated the federal biosurveillance interoperability use-case at the $5^{\text {th }}$ NHIN Forum on December 15, 2008 in Washington, D.C. where Indiana representatives from Regenstrief, MCHD and ISDH demonstrated syndromic surveillance data exchange using the newly designed NHIN gateway. In February 2009 Regenstrief demonstrated the IHE Personnel White Pages (PWP) profile, which provides access to basic human workforce user directory information. Also demonstrated at the Integrating the Healthcare Enterprise (IHE) connect-a-thon in Chicago were the HITSP constructs T63 (Emergency Message Distribution Element Transaction), T64 (Identify Communication Recipients Transaction), C84 (Consult and History \& Physical Note Component), C82 (Emergency Common Alerting Protocol Component), and T81 (Retrieval of Medical Knowledge Transaction). In August 2009, the CDC HIE Collaborative and the CDC demonstrated the biosurveillance situational awareness use case at the CDC-sponsored Public Health Information Network (PHIN) Conference in Atlanta.

\section{Aggregate Summary Data Exchange}

We reviewed the workflow and existing technical systems for identifying and transmitting public health reportable case data including influenza, influenza-like-illness (ILI) and pneumonia. The CDC HIE Collaborative worked closely with CDC stakeholders to create an aggregated data collection format that is used to send data through the NHIN Connect Gateway. This aggregated data collection format is named the Geocoded Interoperable Population Summary Exchange (GIPSE). This format was deployed in September 2009 to send data to the CDC. Its first use was to provide local, state and CDC stakeholders with $\mathrm{H} 1 \mathrm{~N} 1$ surveillance data stratified across 
covariates including age, gender, and geography using a variety of case definitions for ILI and Influenza as defined by HIE and CDC stakeholders.

\section{Delivering Electronic Public Health Alerts to Physicians}

Public health organizations must communicate with clinical care systems for a variety of purposes including case reporting and management, biosurveillance and situational awareness data sharing, and public health alerting. Traditional methods for communicating between public health and clinical entities using telephone, fax, and U.S. postal service often are cumbersome, delayed and inefficient. And although e-mail is a potential option for delivering public-health communications to clinicians, it remains unclear whether e-mails are received in a timely fashion due to several factors including unintentional e-mail filtering, invalid or outdated addresses, and a lack of a reliable mechanism for tracking receipt of such information. To deliver public-health alerts to directly to clinicians in a manner that seamlessly integrates into their workflow, we developed technology that appends public health messages to the existing clinical results delivery service that currently delivers 1.4 million clinical results per month to over 19,000 physicians. We designed, implemented, tested and deployed a public-health alerting system that was first used during the H1N1 influenza outbreak to quickly reach over 3,000 physicians in Marion County. In addition to broadcasting to all physicians, the system can target customized combinations of physician specialties and geographic regions.

\section{Delivering Pre-populated Public Health Reporting Forms to Physicians}

To address physician under-reporting of public health notifiable conditions we developed a prototype system that generates and delivers electronic pre-populated reportable forms to providers using the DOCS4DOCS®clinical messaging system. The system the clinical messaging sends all manner of clinical results to providers who receive them either as a fax, through a designated web-portal or directly into an EHR. The reportable forms system prepopulates the required Indiana reporting forms for reportable diseases and sends the form to physicians at the same time they receive the positive lab result. We hypothesize that because pre-populated forms will reduce the information gathering burden associated with reporting, physician reporting rates will improve when presented with such a form. Future work will evaluate this intervention.

\section{Enhancing Automated Notifiable Condition Detection}

Building on standards for message structure and content (HL7 and LOINC®), the Regenstrief Institute has implemented and maintained an automated notifiable condition reporting system for more than 10 years. The system receives real-time HL7 clinical results from a variety of HIE stakeholders, and automatically translates disparate proprietary codes into standard LOINC codes. It then determines whether the results carried by the message indicate a notifiable condition by checking the abnormal flag sometimes contained in the message, or by comparing the test results with criteria in the PHIN notifiable condition mapping table. We evolved our existing infrastructure to create a modular notifiable condition processor re-usable by other health information exchanges and public health stakeholders. In May, 2009 we were pleased to deliver the Notifiable Condition Detector (NCD) version 1.0 and condition detectors for Shigella, Salmonella and MRSA as an OpenMRS module in both binary and source code formats. The newly enhanced system currently processes up to 300,000 transactions daily and automatically 
detects and transmits up to 450 public health notifiable conditions daily to local and state health departments.

\section{Improving data quality for public health}

Clinical transactions often lack all fields necessary to fully support public health practice. We built and deployed methods within the HIE to enhance the quality of data sent to public health. Two approaches are described below.

Automated LOINC mapping: We worked with the HL7 standards development process to advocate for a new coded element data type (CWE) that permits up to three synonymous vocabulary terms in the HL7 observation identifier field (OBX-3). This enhancement provides greater capacity for sending multiple synonymous code sets, and was necessary to incorporate Logical Observation Identifiers Names and Codes (LOINC ${ }^{\circledR}$ ) into HIE transactions. To standardize HIE data for public health uses, we deployed an ongoing process to automatically transform non-standard inbound codes into LOINC ${ }^{\circledR}$ codes.

Enhancing Provider data: Because identifying physician information helps speed public health case management processes, we leveraged a master provider file to enhance incomplete provider information for incoming message streams. This process increases completeness of the provider telephone number by more that $40 \%$. Providing MCHD with this information allows epidemiologists to perform case management functions more efficiently.

\section{Conclusions}

As this work continues, we will advance the quality and quantity of clinical data and will seek methods for providing public health personnel with increasingly seamless and direct access to the HIE data repository for approved purposes. After completing provider enhancement, we will focus on enhancing patient data using the HIE global patient registry. This will allow public health to have complete patient information for case reporting. These and other technological advancement will be the benchmark for a HIE to send clinical data to public health and the CDC through the NHIN.

The longstanding, successful Indiana coalition has developed leading-edge technologies that clearly demonstrate the feasibility and value of leveraging HIE to support a variety of public health use cases. By re-using HIE for public health purposes we have made substantive meaningful improvements in the quality and quantity of clinical data that is currently being exchanged with public health. Specifically, provider information that is often missing or incomplete has been improved so public health doesn't have to search for the information. Further, by participating in demonstrations at the IHE, HIMSS and PHIN annual conferences, we were able to highlight the biosurveillance detection and monitoring scenarios to various audiences. Moreover, using public health alerting and pre-populated forms as prototypes, we have enabled rapid and seamless bidirectional communication between public health and clinical care systems. This framework presents a host of new opportunities to increasingly support public health practice. By connecting stakeholders from clinical care and public health organizations through comprehensive, sustained activity, we will advance the digital channels 
and trust relationships that are necessary to establish the next generation of infrastructure that will meaningfully support increasingly complex public health processes.

\section{Conflicts of interest}

The authors have no conflicts of interest to report at this time.

\section{Acknowledgments}

This project was funded by the Centers for Disease Control \& Prevention under contract 2002008-24368. The content of this publication does not necessarily reflect the views or policies of the Department of Health and Human Services, nor does mention of trade names, commercial products, or organizations imply endorsement by the U.S. Government.

\section{Correspondence}

Shaun J. Grannis, MD, MS, FAAFP

sgrannis@ regenstrief.org 